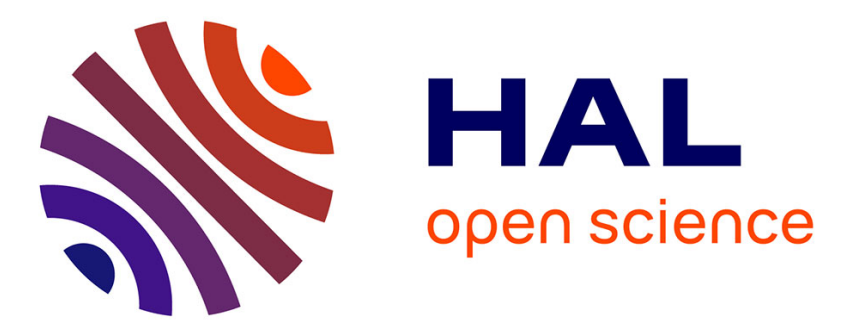

\title{
Increasing the Sensitivity and Selectivity of Metal Oxide Gas Sensors by Controlling the Sensitive Layer Polarization
}

Nicolas Dufour, Yoan Veyrac, Philippe Menini, Frédéric Blanc, Chabane Talhi, Bernard Franc, Christian Ganibal, Corinne Wartelle, Khalifa Aguir

\section{To cite this version:}

Nicolas Dufour, Yoan Veyrac, Philippe Menini, Frédéric Blanc, Chabane Talhi, et al.. Increasing the Sensitivity and Selectivity of Metal Oxide Gas Sensors by Controlling the Sensitive Layer Polarization. IEEE Sensors 2012, Oct 2012, Taipei, Taiwan. 4p. hal-00732255

\section{HAL Id: hal-00732255 https://hal.science/hal-00732255}

Submitted on 14 Sep 2012

HAL is a multi-disciplinary open access archive for the deposit and dissemination of scientific research documents, whether they are published or not. The documents may come from teaching and research institutions in France or abroad, or from public or private research centers.
L'archive ouverte pluridisciplinaire HAL, est destinée au dépôt et à la diffusion de documents scientifiques de niveau recherche, publiés ou non, émanant des établissements d'enseignement et de recherche français ou étrangers, des laboratoires publics ou privés. 


\section{Increasing the Sensitivity and Selectivity of Metal Oxide Gas Sensors by Controlling the Sensitive Layer Polarization}

\author{
N. Dufour, Y. Veyrac, P. Menini, F. Blanc, C. Talhi, B. \\ Franc and C. Ganibal \\ LAAS-CNRS \\ Toulouse, France \\ ndufour@laas.fr \\ P. Menini \\ Paul Sabatier University \\ Toulouse, France
}

\begin{abstract}
The effects of bias current on sensitivity and selectivity of resistive Metal-Oxide (MOX) sensors toward gases have been investigated. While the working temperature is kept constant, it has been found that tuning the polarization of the MOX thin film induces changes on its sensitivity toward gases. Besides, the behavior of sensitivity versus bias current depends on the nature of the gas and of the thin sensing film, meaning it is possible to enhance the selectivity by adjusting the polarization of different sensitive layers. This underutilized parameter provides a new way of improving easily gas sensors sensitivity and above all selectivity. It would then be interesting to polarize individually each sensor of a multi-sensor device in order to optimize the whole system performances, which may represent a breakthrough in the development of smart E-Nose.
\end{abstract}

\section{INTRODUCTION}

Metal oxide thin film sensors have been widely used for gas sensing applications thanks to their sensitivity toward a large variety of gases [1]. The main reaction occurring at the MOX thin film surface is the ionization of adsorbed oxygen, creating a depletion layer at the grain boundaries $[2,3]$. The sensing mechanism is based on the gas reaction related modulation of the as created Schottky barriers, thus changing the resistivity of the thin film [4]. Considering all the rectifying junctions, the current/voltage characteristic of MOX films exhibits a non-linear behavior, meaning that their resistance depends on the polarization [5, 6]. Sensitivity toward gases, which is closely related to these phenomena, also turns out to be polarization dependant [7]. The influence of the sensitive layer biasing on sensitivity to carbon monoxide has been observed for a couple of devices yet $[8,9$, 10], underlining distinct behaviors for different metal oxides. However, polarization of MOX layers still suffers from a lack of investigations even though it seems to be of great interest in

\author{
N. Dufour, C. Wartelle \\ DiMat \\ Renault S.A.S. \\ Guyancourt, France \\ K . Aguir \\ IM2NP - UMR 7334 \\ CNRS \\ Marseille, France
}

order to enhance gas sensor performances. The work presented here aims at characterizing the influence of this parameter so as to improve both the sensitivity and selectivity of MOX sensors.

\section{THEORICAL}

Between 100 and $500^{\circ} \mathrm{C}$, the interaction with oxygen involves its ionosorption in atomic and molecular form [3]. The oxygen chemisorption equation is the following:

$$
(\beta / 2) \mathrm{O}_{2}^{\text {gas }}+\alpha \cdot \mathrm{e}^{-}+\mathrm{S} \leftrightarrow \mathrm{O}_{\beta \mathrm{S}}^{-\alpha},
$$

where $\mathrm{O}_{2}^{\text {gas }}$ is an oxygen molecule, $e^{-}$is an electron, $S$ is an unoccupied chemisorption site, $\mathrm{O}_{\beta \mathrm{S}}^{-\alpha}$ is a chemisorbed oxygen species, and $\alpha$ and $\beta$ are equal to 1 or 2 for singly or doubly ionised form and for atomic or molecular form respectively.

The nanoparticle-made MOX thin films model consists of strings of grains, where the conduction is limited by the grain boundary voltage barriers [2]. The conductance can thus be expressed as:

$$
\mathrm{G}=g q \mu_{\mathrm{S}} \mathrm{N}_{\mathrm{d}} \exp \left(-q \mathrm{~V}_{\mathrm{s}} / \mathrm{kT}\right),
$$

where $G$ is the conductance, $g$ is a constant determined by the geometry, $q$ is the elementary charge, $\mu_{s}$ is the electron mobility, $N_{d}$ is the density of donors and $V_{s}$ the potential barrier. 


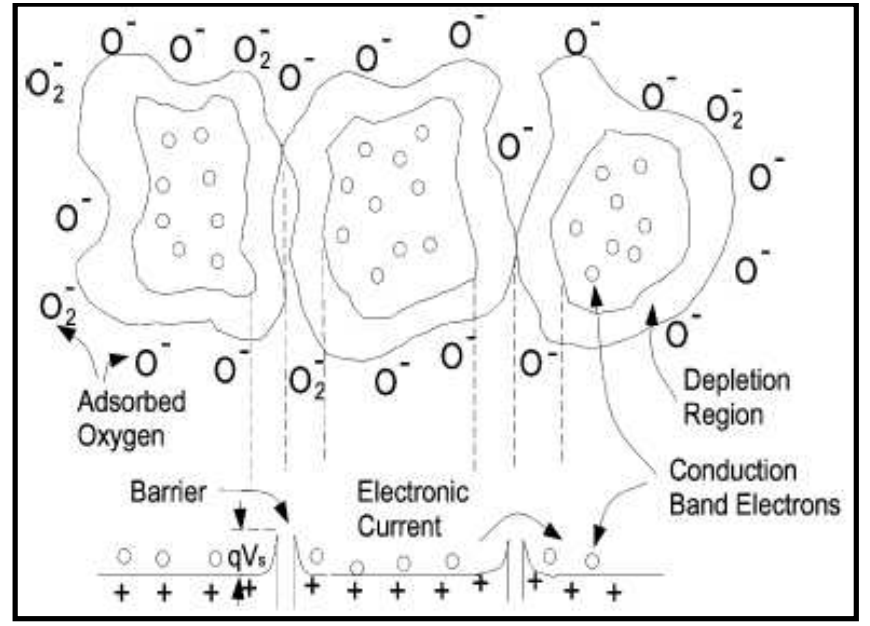

Figure 1. Model of grain boundary of a MOX thin film

The density of surface states depends on the polarization as described in [7]; the developed model is grounded on the energy band theory. The reaction between a gas and the ionized MOX layer is related to the surface state occupancy. The variation of the potential barrier height occurring during the reaction is determined by the previous phenomenon. According to (2), the conductance varies with Vs, so the sensitivity is directly linked to the polarization.

\section{EXPERIMENTAL}

\section{A. Devices}

The tested devices have been developed on an optimized microhotplate that can work at high temperature and low power consumption $\left(500^{\circ} \mathrm{C}, 55 \mathrm{~mW}\right)$ (Fig. 2). The platform consists of a silicon bulk on which a thermally resistive bilayer $\mathrm{SiO}_{2} / \mathrm{SiN}_{\mathrm{x}}$ membrane was grown. Afterwards, Pt metallization was realized by lift-off to define simultaneously a heating resistor and the electrodes of a sensing resistor. Contacts were opened in a previously deposited passivation layer. Finally, the rear side of the bulk was etched to release the membrane in order to increase the thermal resistance and then to limit thermal dissipation. It is then possible to deposit a metal-oxide layer to form the sensing thin film resistor. Characteristics of the tested MOX layers are specified in Table 1.

TABLE I. SENSITIVE LAYER SPECIFICATIONS

\begin{tabular}{|l|lr|c|c|}
\hline \multicolumn{1}{|c|}{$\begin{array}{c}\text { Metal } \\
\text { Oxide }\end{array}$} & \multicolumn{2}{|c|}{ Deposit method } & $\begin{array}{c}\text { Annealing } \\
\text { Temperature }\end{array}$ & $\begin{array}{c}\text { Operating } \\
\text { Temperature }\end{array}$ \\
\hline $\mathrm{WO}_{3}$ & $\begin{array}{l}\text { Magnetron Reactive } \\
\text { Sputtering }\end{array}$ & $350^{\circ} \mathrm{C}$ & $300^{\circ} \mathrm{C}$ \\
\hline $\mathrm{ZnO}$ & $\begin{array}{l}\text { Magnetron Reactive } \\
\text { Sputtering }\end{array}$ & $550^{\circ} \mathrm{C}$ & $500^{\circ} \mathrm{C}$ \\
\hline $\mathrm{SnO}_{2}$ & Inkjet & $550^{\circ} \mathrm{C}$ & $500^{\circ} \mathrm{C}$ \\
\hline $\begin{array}{l}\mathrm{SnO} 2 \mathrm{Pt}- \\
\text { doped }\end{array}$ & Inkjet & $550^{\circ} \mathrm{C}$ & $500^{\circ} \mathrm{C}$ \\
\hline
\end{tabular}

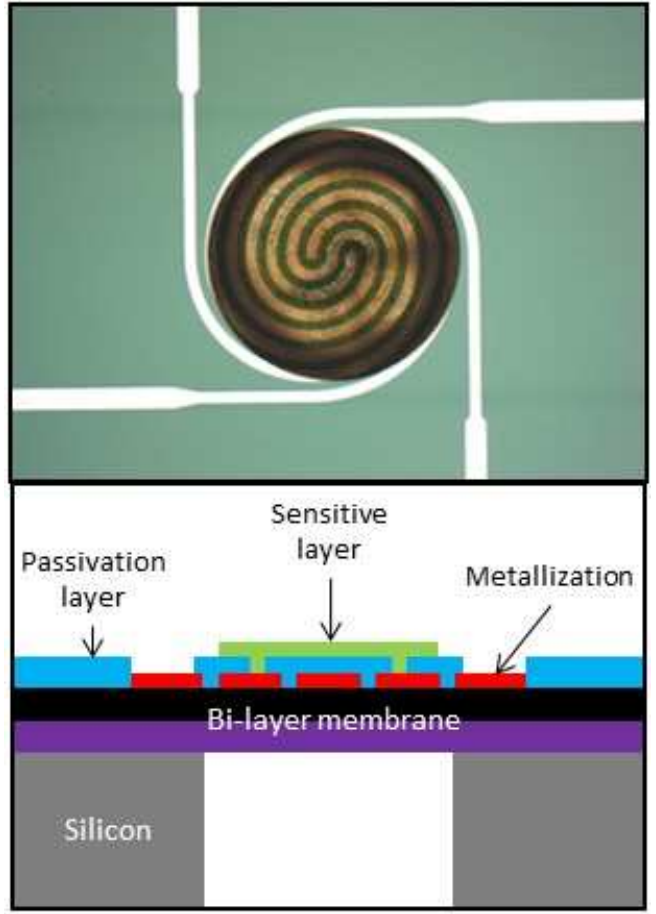

Figure 2. Photo and cross-sectional view of the fabricated gas sensor

\section{B. Test bench}

The sensors are placed into a chamber where gas is flowing (Fig. 3). The composition and humidity of the gas mixture are controlled by Mass Flow Controllers and the global flow rate is checked with a debimeter. The heating and the sensing resistors are connected to two Source Measurement Units (Keithley 2400). The whole test bench is automatically controllable thanks to a suitable interface and a dedicated software.

\section{Experimental process}

The heating resistor biasing voltage is set prior to the test and kept constant afterwards, ensuring a constant working temperature. As for the sensing layer, the most classical method of measurement consists in applying a bias voltage while the current is measured. Both this technique and the dual one (applied current and measured voltage) have been tested and compared. No matter how the polarization is applied, no significant difference has been found in the behavior of sensitivity versus the polarization power (Fig. 4), thus the measurements were done with a bias current so as to address embedded applications. Two measurement protocols were then considered. The first one consists in realizing the reaction with gas for each bias current value. After a period of stabilization under dry air (at least one hour), the specific gas is added during a given time while ensuring the global flow remains constant. Finally, the aforementioned gas injection is stopped to let the sensor recover under dry air. 


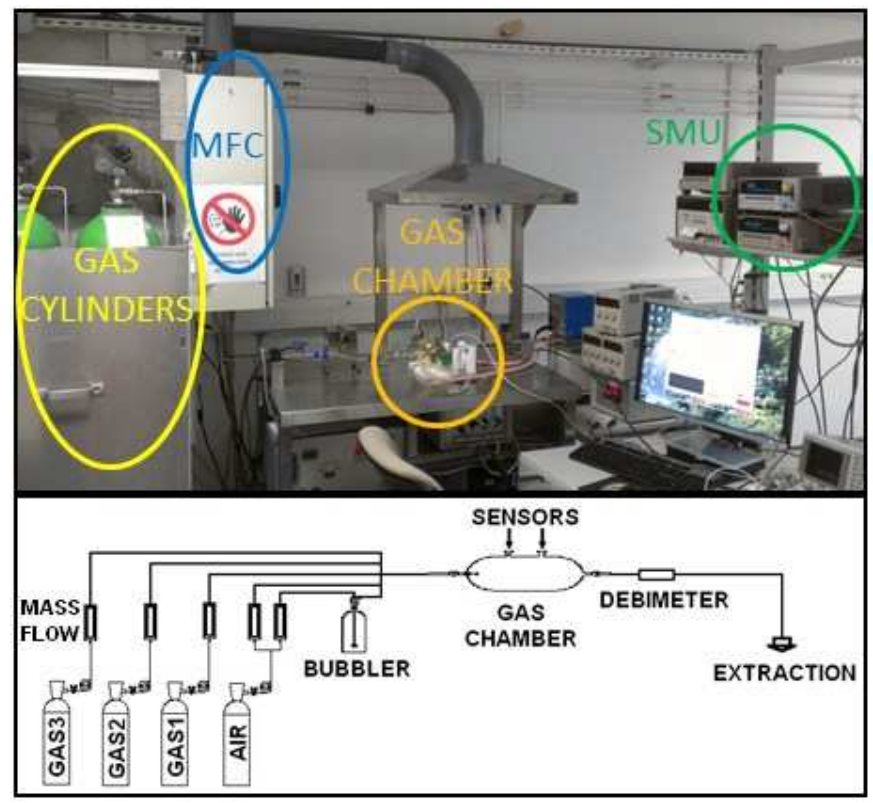

Figure 3. Photo and diagram of the test bench

For the second method, the sensor is stabilized under dry air at a particular bias current and then a sweep is realized to cover the desired range of polarization (waiting a few minutes for stabilization at each bias current value). Only then the gas is injected, and after stabilization the same bias current sweep as before is operated, to obtain the values of resistance under gas. The two protocols lead to the same sensitivities (Fig. 4), so the second one which allows faster measurement sessions is preferred.

Responses of four MOX layers to three gases (CO200ppm, $\mathrm{NO}_{2}-10 \mathrm{ppm}$, and $\mathrm{NH}_{3}-10 \mathrm{ppm}$ ) were characterized at different bias currents, ranging from 0.1 to $10 \mu \mathrm{A}$, and the corresponding sensitivities were compared.

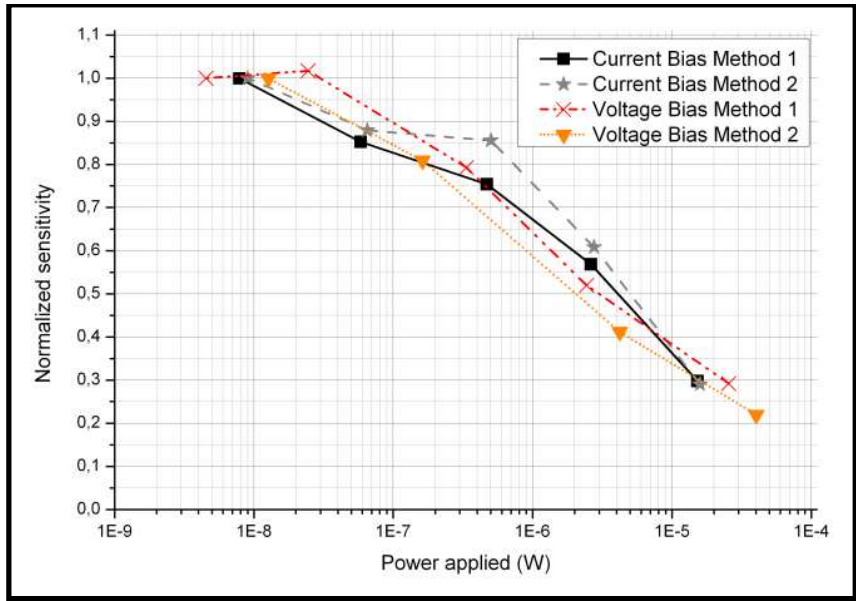

Figure 4. Normalized sensitivities to $\mathrm{NO}_{2} 10 \mathrm{ppm}$ with $\mathrm{WO}_{3}$ sensing layer for different polarization methods

\section{Results and discussion}

The sensitivity is calculated as the ratio of the absolute difference between the stabilized resistances of the device under dry air and under the specific gas to the resistance under dry air:

$$
S=\left|R_{\text {air }}-R_{\text {gas }}\right| / R_{\text {gas }}
$$

It is defined for each bias current and then normalized in order to emphasize the general trend of the variation of sensitivity with polarization and to make the results for different MOX layers and different gases comparable. The lowest bias current value, namely $100 \mathrm{nA}$, has been chosen as the reference for the normalization and the corresponding sensitivity is then set to +1 or -1 , according to the following formula:

$$
\mathrm{S}_{\mathrm{i}} \text { norm }=+/-\left(1-\left(\mathrm{S}_{100 \mathrm{nA}}-\mathrm{S}_{\mathrm{i}}\right) / \mathrm{S}_{100 \mathrm{nA}}\right) .
$$

The sign plus is used for an oxidizing type reaction, meaning the response mechanism results in an increase of the resistance, whereas the sign minus corresponds to a reducing type reaction. This shaping enables the comparison of the sensitivity evolution with increasing bias currents for all the available sensing layers and gases.

The responses of the four layers to carbon monoxide $(\mathrm{CO})$ were recorded and the corresponding sensitivities were calculated (3) and compared for different bias currents (Fig.5).

The $\mathrm{ZnO}$ sensor shows a steady sensitivity to $\mathrm{NO}_{2}$ whereas its response to $\mathrm{NH}_{3}$ underlines a huge increase of sensitivity with stronger bias currents (Fig.6). As for $\mathrm{CO}$, the behavior of the $\mathrm{ZnO}$ layer changes completely since the response has an oxidizing type for the lowest bias currents and a reducing type for the highest one. Such a change turns out to be of primary importance as far as the tuning of selectivity is concerned.

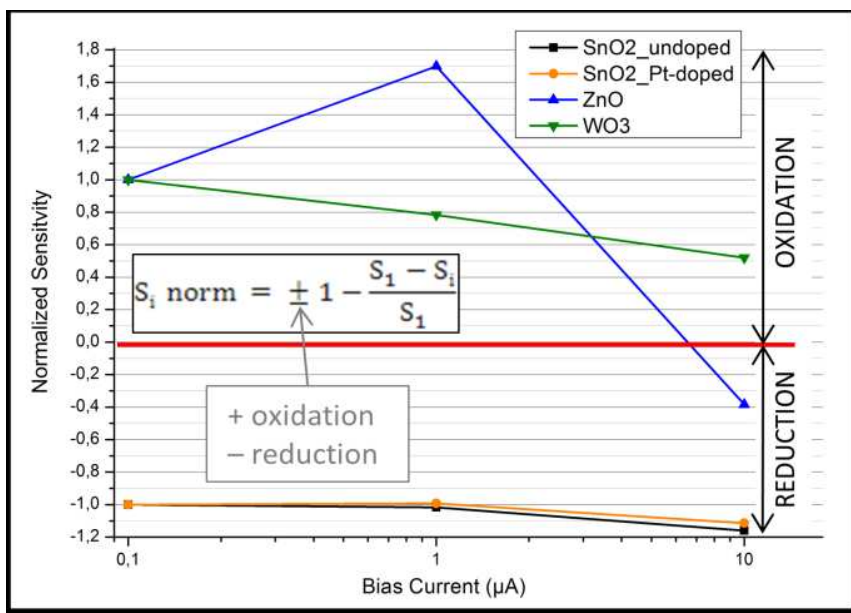

Figure 5. Normalized sensitivities of four sensitive layers to CO $200 \mathrm{ppm}$ 
The general behavior of the $\mathrm{SnO}_{2}$ sensor is the exact opposite. Indeed, its sensitivity to $\mathrm{CO}$ and $\mathrm{NH}_{3}$ remains almost constant whereas the sensitivity to $\mathrm{NO}_{2}$ drops at high bias current.

The $\mathrm{CO}$ has an oxidizing behavior on the $\mathrm{WO}_{3}$ sensor, with a variable sensitivity (Fig. 7). The $\mathrm{NO}_{2}$ sensitivity of this sensor decreases strongly with higher bias currents, from $1500 \%$ at $100 \mathrm{nA}$ to $400 \%$ at $10 \mu \mathrm{A}$. The opposite trend is observed for the $\mathrm{NH}_{3}$, with a sensitivity multiplied by more than two between $100 \mathrm{nA}$ and $10 \mu \mathrm{A}$.

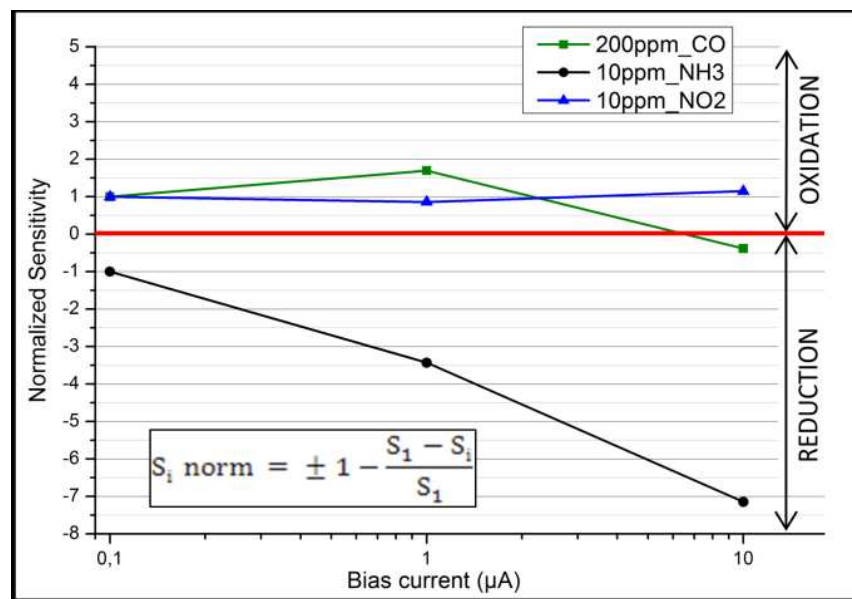

Figure 6. Normalized sensitivities of $\mathrm{ZnO}$ sensitive layer to three different gases

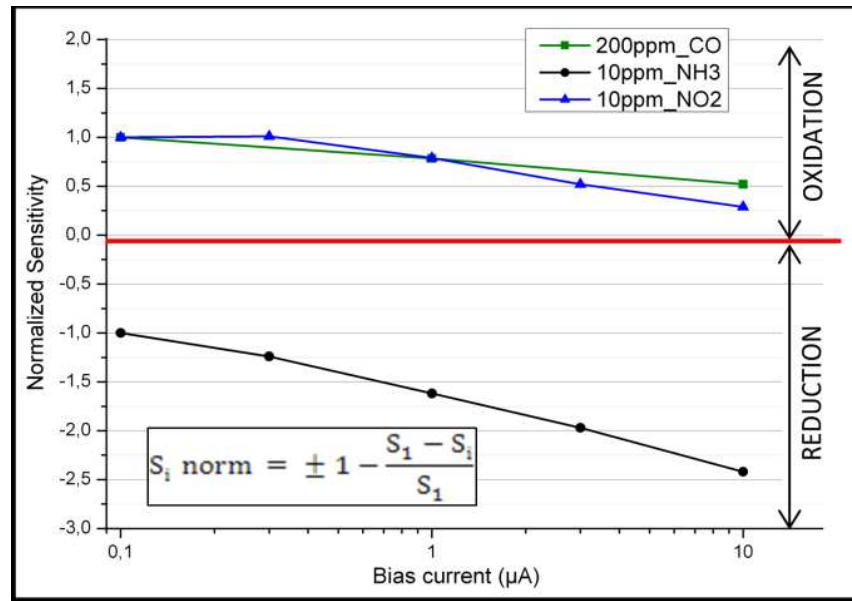

Figure 7. Normalized sensitivities of $\mathrm{WO}_{3}$ sensitive layer to three different gases

\section{CONCLUSION}

Polarization of the sensing layer has been inspected for three distinct gases with four different sensing layers. The obtained results, for only one heater temperature of each sensing layer, show a highly dependent response to gases according to the polarization. The influence of humidity has been investigated and it turns out that the effects of polarization on sensitivity are significant under different relative humidity values. As for the temperature impact, the $\mathrm{WO}_{3}$ sensing layer has been tested under $\mathrm{NH} 3-10 \mathrm{ppm}$ with two different operating temperatures $\left(200\right.$ and $\left.300^{\circ} \mathrm{C}\right)$, revealing opposite behaviors. Indeed, at the lowest temperature, the sensitivity decreases strongly when the bias current is increased, and conversely for the highest one. Combining the effects of the sense and the heater polarizations, which are post-process tunable parameters, enables to modulate the sensitivity of each sensor to each specific gas. That provides many possibilities to enhance the selectivity; a highly discriminating multi-sensor could then be realized.

\section{REFERENCES}

[1] C. Wang, L. Yin, L. Zhang, D. Xiang and R. Gao, "Metal oxide gas sensors: sensitivity and influencing factors," Sensors, vol. 10, pp. 20882106, 2010.

[2] J. Ding, T.J. McAvoy, R.E. Cavicchi and S. Semancik, "Surface state trapping models for $\mathrm{SnO}_{2}$-based microhotplate sensor," Sensors And Actuators B, vol. 77, pp. 597-613, 2001.

[3] N. Barsan and U. Weimar, "Conduction model of metal oxide gas sensors," Journal of Electroceramics, vol. 7, pp. 143-167, 2001.

[4] N. Barsan and U. Weimar, "Understanding the fundamental principles of metal oxide based gas sensors; the example of $\mathrm{CO}$ sensing with $\mathrm{SnO} 2$ sensors in the presence of humidity," Journal of Physics: Condensed Matter, vol. 15, pp. 813-839, 2003.

[5] Y. Min, "Properties and sensor performance of zinc oxide thin films," $\mathrm{PhD}$ in Electronic, Photonic and Magnetic Materials, Massachusetts Institute Of Technology, September 2003.

[6] V.V. Simakov, O.V. Yakusheva, A.I. Grebennikov and V.V. Kisin, "Current-voltage characteristics of thin-film gas sensor structures based on tin dioxide," Technical Physics Letters, vol. 31, pp. 339-340, 2005.

[7] A. Varpula, S. Novikov, J. Sinkkonen and M. Utriainen, "Bias dependent sensitivity in metal-oxide gas sensors," Sensors And Actuators B, vol. 131, pp. 134-142, 2008.

[8] S.M.A. Durrani, "Biasing voltage dependence of sensitivity of electron beam evaporated $\mathrm{SnO}_{2}$ thin film CO sensor," Sensors, vol. 6, pp. 11531160,2006

[9] S.M.A. Durrani and M.F. Al-Kuhaili, "Effect of biasing voltages and electrode metals and materials on the sensitivity of electron beam evaporated $\mathrm{HfO} 2$ thin film $\mathrm{CO}$ sensor," Materials Chemistry and Physics, vol. 109, pp. 56-60, 2008.

[10] S.M.A. Durrani, M.F. Al-Kuhaili, I.A. Bakhtiari and M.B. Haider, "Investigation of the carbon monoxide gas sensing characteristics of tin oxide mixed cerium oxide thin films," Sensors, vol. 12, pp. 2598$2609,2012$. 